\title{
Extension of FRI for Modeling of Electrocardiogram Signals
}

\author{
R. Frank Quick, Senior Member, IEEE, Ronald E. Crochiere, Fellow, IEEE, John H. Hong, Ali \\ Hormati, Member, IEEE, and Gilles Baechler
}

\begin{abstract}
Recent work has developed a modeling method applicable to certain types of signals having a "finite rate of innovation" (FRI). Such signals contain a sparse collection of time- or frequency-limited pulses having a restricted set of allowable pulse shapes. A limitation of past work on FRI is that all of the pulses must have the same shape. Many real signals, including electrocardiograms, consist of pulses with varying widths and asymmetry, and therefore are not well fit by the past FRI methods. We present an extension of FRI allowing pulses having variable pulse width (VPW) and asymmetry. We show example results for electrocardiograms and discuss the possibility of application to signal compression and diagnostics.
\end{abstract}

\section{INTRODUCTION}

Recent work [1, 2] has developed a modeling method applicable to certain types of signals having a "finite rate of innovation" (FRI). Such signals contain a sparse collection of time- or frequency-limited pulses having a restricted set of allowable pulse shapes. The original FRI theory [1, 2] was applicable only to signals consisting of certain types of pulses with a fixed width, such as bandlimited delta-functions. These are a good model for certain signals, such as multipath channel impulse responses when the channel consists of a few well-separated delays. Other signals, like the electrocardiogram (ECG), consist of pulses with a wide range of pulse widths and asymmetries, sometimes partially overlapping, and are not well fit by a model which includes only a single pulse shape.

We have developed an extension of FRI which we call Variable Pulse Width (VPW) FRI, in which pulses of varying widths and asymmetries can be analyzed using the mathematical treatment of FRI. The usefulness of this analysis for the ECG is that the signal can be represented accurately using only a small number of coefficients, resulting in compression and potentially noise reduction relative to the original data. With the asymmetric component included, the fit to actual data should be improved relative to models based solely on symmetric pulses such as Gaussians. It can also be hoped that future work will determine ways to use the coefficients directly in diagnosis.

R. F. Quick, R. E. Crochiere and J. H. Hong are with Qualcomm Incorporated, San Diego, CA 92121 USA (phone: 858-658-3608; fax: 858651-5180; e-mail: fquick@qualcomm.com, rcrochie@qualcomm.com, johnhong@qualcomm.com).

A. Hormati is with EPFL, Lausanne, Switzerland, and was formerly Visiting Faculty at Qualcomm Incorporated (e-mail: ali.hormati@epfl.ch).

G. Baechler is with EPFL, Lausanne, Switzerland, and was formerly an Engineering Intern at Qualcomm Incorporated (e-mail: gilles.baechler@epfl.ch)

\section{FRI BACKGROUND}

FRI is a sparse sampling theory that works on continuous time signals consisting of sparse components with certain functional forms. FRI can also be considered an extension of system identification theory, which originated with Prony [3] back in 1795 . The bulk of work in this area has been carried out more recently, mainly beginning in the 1980s when computing resources that could carry out the required analysis became commonplace. The FRI formulation is a new development introduced by Vetterli, Marziliano and Blu [1], and has been well summarized by Blu et al. [2].

\section{A. Noiseless Signals}

Prony's work showed that if a signal consists of a sum of complex exponentials, it is possible to determine the components of the signal through the solution of a set of linear equations. The argument, briefly, is that the exponential components are the homogeneous solutions of a finite difference equation, i.e., they are the roots of the characteristic polynomial of the difference equation. The difference equation, applied to the signal, always produces zero, therefore the equation can be identified by solving the homogeneous equation

$$
\boldsymbol{X} d=0
$$

where $\boldsymbol{X}$ is a square Toeplitz matrix formed from the signal data and $d$ is a vector containing the coefficients of the difference equation. This equation is most easily solved by singular value decomposition (SVD) of $\boldsymbol{X}$ taking $d$ as a right eigenvector having a singular value of zero.

Following Blu et al. [2] we call $d$ an annihilator for $\boldsymbol{X}$, since the product is zero. The characteristic polynomial of the corresponding difference equation is also called the annihilator polynomial. The roots of the annihilator polynomial determine the exponential components of the signal. The amplitudes of these components can be determined by solving another set of linear equations.

\section{B. Noisy Signals}

In the case where the signal is noisy, one typically obtains more samples than required for the solution, making $\boldsymbol{X}$ a "tall" matrix with more rows than columns. In that case the best solution for $d$ in the total least-squares sense can also be found by the SVD approach, taking $d$ as the right eigenvector having the smallest singular value. This approach is similar to that discussed by Pisarenko [4].

The optimal approach, however, is to solve a constrained least-squares problem that retains the structure of the underlying model. De Moor [6] stated the problem in this way:

$$
\min _{b, y}\|a-b\|^{2} \text { s.t. } \boldsymbol{B} y=0 \text { and }\|y\|=1
$$


where $a$ is the input data vector; $b$ is the estimate of the data; $\boldsymbol{B}$ is a Toeplitz matrix (generally not square) formed from $b$ in the manner described by Blu et al. [2]; and $y$ is the annihilator for $\boldsymbol{B}$. This can be stated in words as "find the vector generated by a difference equation of fixed order that best approximates the signal vector." Alternatively the problem can be seen as finding the best autoregressive (AR) model for the data in the sense of minimum squared error.

De Moor [6] provided an efficient iterative solution of (1) using Lagrange multipliers. Unfortunately De Moor's algorithm does not always converge. An alternative is the fmincon() function of the Matlab ${ }^{\circledR}$ Optimization Toolkit, which includes an interior-point algorithm suitable for this problem. This algorithm appears to always succeed in finding a solution (which may be only a local minimum) but its execution is slow.

Blu et al. [2] describe a faster but suboptimal procedure based on the iterative "cleaning" algorithm described by Cadzow [7]. (Note that algorithms similar to Cadzow's were described earlier by Tufts and Kumaresan [8].) The FRI analysis procedure of Blu et al. can be summarized as follows:

- Use the Cadzow algorithm to find an approximate annihilator polynomial for the matrix $\boldsymbol{X}$.

- Determine the roots of the annihilator polynomial. These are the estimated exponential components of the signal.

- Use least-squares regression to determine the (complex) amplitudes of the exponential components forming the estimate of the signal.

Although the Cadzow algorithm is suboptimal, we generally find that the signal estimate is within a dB of the optimal solution as returned by fmincon(). Thus it provides a useful technique for practical problems.

The Cadzow algorithm uses iterated SVD, but it is fast enough to be used with ECG data, which are of low bandwidth. Perhaps the most practical algorithm, however, is one based on ESPRIT [5] because it computes the exponential components in a single pass using two eigenvector decompositions, avoiding the iterations needed by the Cadzow algorithm. Hormati [9] provides a description of an ESPRIT-based algorithm that can be used for FRI.

\section{FRI Signal Modeling}

In addition to showing how to obtain a practical solution for noisy signals, Blu et al. [2] discuss conditions under which the solution provides compression of the data. For example, if the time-domain signal consists of bandlimited Dirac $\delta$-functions with arbitrary times of arrival, the FFT of the signal will consist of complex exponentials of constant envelope over the bandwidth of the signal. FRI analysis applied to the FFT of the signal can then yield the locations and amplitudes of the delta-functions with an accuracy finer than the time-domain sampling interval.

Vetterli et al. [1] showed that FRI can include other pulse shapes, including Gaussians and other special cases such as splines. However, none of these pulse types alone is efficient for modeling signals that contain pulses of varying widths and shapes.

\section{THE ECG SIGNAL}

One heartbeat from a sample ECG signal is shown in Fig. 1:

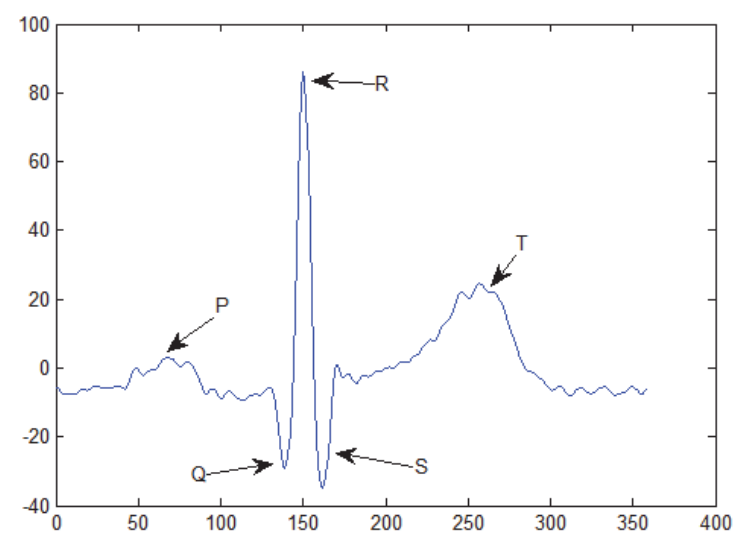

Figure 1. Example ECG

The ECG normally contains five pulses, usually labeled PQRST. In this figure the P pulse is of low amplitude and is somewhat obscured by the noise. The central QRS complex is clearly seen and is both larger in amplitude and much narrower than either $\mathrm{P}$ or $\mathrm{T}$. The T pulse is wide and seems to be somewhat asymmetric. The original signal was sampled at $360 \mathrm{~Hz}$; it has been interpolated to a $1440 \mathrm{~Hz}$ sampling rate for this figure.

The main point to be taken from this figure is that no single pulse shape can be used to fit all five pulses. The variable pulse-width variant developed by the authors will be seen to fit the data quite well with a small number of pulses, unlike a signal model obtained from the Dirac delta-functions used in the original FRI.

\section{VARIABLE PUlse-WIDTH FRI}

Prony's formulation of the problem included signal components that could be any complex exponential function of time or frequency. For the ECG, the time-domain signal consists of a small number of isolated pulses, so the Fourier transform domain is likely to contain approximately exponential signals. The general form of an exponential signal in the frequency domain is:

$$
F(\omega)=\gamma e^{\alpha \omega}
$$

where the exponential factor $\alpha$ and the amplitude $\gamma$ are, in general, complex numbers. But since the ECG is a realvalued signal, the Fourier transform must satisfy $F(-\omega)=$ $F^{*}(\omega)$, so a more appropriate functional form is

$$
F(\omega)= \begin{cases}\gamma e^{-R e(\alpha) \omega} e^{-j \operatorname{Im}(\alpha) \omega}, & \omega>0 \\ \gamma^{*} e^{\operatorname{Re}(\alpha) \omega} e^{-j \operatorname{Im}(\alpha) \omega}, & \omega<0\end{cases}
$$

The algorithm described by Blu et al. [2] performs the FRI analysis using both the positive and negative frequencies, symmetrically distributed about $\omega=0$. But we can see from (2) that the Fourier transform is not truly 
exponential over all frequencies unless $\operatorname{Re}(\alpha)=0$ and $\gamma$ is real. In practice we find that using symmetrically placed positive and negative frequency components almost always produces unit-circle roots for the annihilator polynomial, hence the signal approximation in the time domain consists only of Dirac delta-functions. These pulses can be widened by assuming a limited bandwidth, but all the pulses will have the same width, determined by the bandwidth.

Our first step in obtaining a more general pulse shape is, therefore, to perform the FRI analysis using only the positive (or negative) frequencies, an interval over which the Fourier transform can be truly exponential for any complex exponent and coefficient values. We can use the same FRI algorithm to find the components of the signal and then reproduce the signal by summing the inverse Fourier transforms of (1) for the signal components. Each inverse transform has two parts, one corresponding to the real part of $\gamma$ and another to the imaginary part. To simplify the notation, let $\operatorname{Re}(\alpha) \triangleq \sigma$ and $\operatorname{Im}(\alpha) \triangleq \tau$. Then:

$$
f(t)=\operatorname{Re}(\gamma) \frac{2 \sigma}{\sigma^{2}+(t-\tau)^{2}}-\operatorname{Im}(\gamma) \frac{2(t-\tau)}{\sigma^{2}+(t-\tau)^{2}}
$$

The two terms are seen to be a symmetric pulse of Lorentzian, also called Cauchy, form; and an asymmetric pulse which is proportional to $(t-\tau)$ times the symmetric pulse shape.

Equation (3) is, of course, a continuous-time signal of infinite support, whereas in practice we use the Discrete Fourier Transform (DFT) of a sampled and time-limited version of the signal. The DFT, like a Fourier series, assumes that the signal is periodic with the period equal to the signal duration.

Since FRI finds exponential signal components in the DFT, the discrete model in frequency is simply a sampled version of (2). For the signal in the time domain, the inverse DFT of a sampled version of (2) would give a periodic version of (3) which can be used to reconstruct the signal. We find that it is equally practical to reconstruct the signal in the frequency domain using the sampled version of (2) and then take the inverse DFT to obtain the estimated time signal.

Baechler [10] has also implemented a routine to process ECG recordings containing several heart beats. Since VPWFRI is a DFT-based algorithm, the first step is to extract individual heartbeats from the complete data. This is achieved using the QRS detection algorithm described in [11]. Each beat is then processed using the procedure described above and reconstructed with the parameters obtained. Finally the results are stitched back together. In order to have a smooth transition between the recovered beats, the buffers holding input beats overlap in time. The reconstructions are then stitched together using a taperedcosine (Tukey) window which smoothes the boundaries.

\section{RESUltS}

Fig. 2 shows the original signal and its reconstruction using VPW-FRI with an assumed signal order of seven, using the interior-point algorithm of fmincon() to find the optimal annihilator polynomial. ${ }^{1}$ The order seven is found by experiment to give good results for most ECG signals. Further study is needed to determine how best to decide the model order for all such signals.

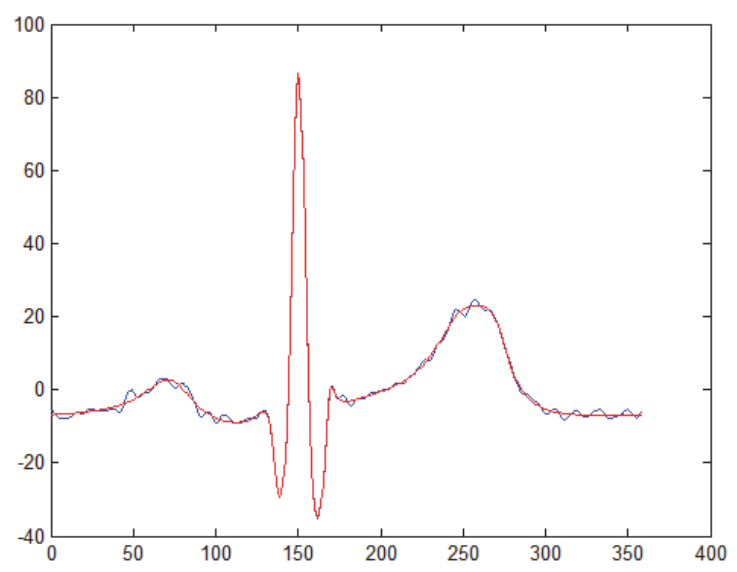

Figure 2. Original (blue) and reconstructed (red) ECG

It can be seen that the reconstruction preserves the major features of the waveform while "cleaning" most of the smaller, narrower features that are due to noise. It can be said that the VPW-FRI algorithm is functioning as a model-based noise reduction process.

Fig. 3 adds stem plots to Fig. 2 to show the timing and amplitude of the symmetric and asymmetric components of the reconstruction:

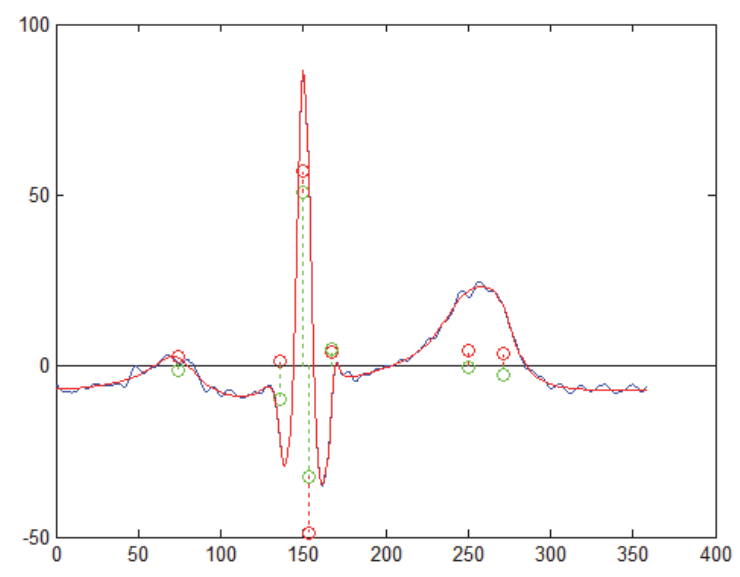

Figure 3. Plot from Fig. 2, with stems showing the location and amplitude of the symmetric (red) and asymmetric (green) components

Here we can see that using order seven for the model gives the algorithm two more degrees of freedom than the number of visible pulses, and these pulses are placed so as to better fit the signal. In this example, an extra pulse is found in the $\mathrm{R}$ and $\mathrm{T}$ pulses, perhaps improving the fit to the

\footnotetext{
${ }^{1}$ Since fmincon() may return a local minimum, this algorithm must be invoked several times with different initial conditions to improve the probability that the solution is indeed the global optimum.
} 
underlying physical signals that are not exactly of the form of (3).

In other samples, we sometimes observe the extra pulses introducing short pulses that model some aspect of the noise. Indeed, in noisy samples we generally find that there are a number of locally-optimal fits that occur when the initial conditions are varied. These locally optimal variations usually have similar overall SNR for the reconstruction; it matters little which minor signal aspect the algorithm includes in its model. Of course, if there is a high level of noise, this algorithm will perform poorly because there are too many "pulses", most of which are not part of the underlying signal.

This example shows that VPW-FRI does not reconstruct the signal with a one-to-one relationship between the visible pulses in the signal and the variable-width pulses used in the reconstruction. It can therefore be stated that although the model computed by VPW-FRI fits the signal as well as possible under the criterion (1), it is a purely mathematical treatment without direct relationships to the underlying physics nor physiology. A challenge for future research is to determine whether the VPW-FRI model can be translated into physiological descriptions of the signal, and vice versa. Such a translation could enable both improved simulations of the ECG and diagnostic interpretation using VPW-FRI.

\section{CONCLUSION}

The order seven VPW-FRI model fits the data quite well. It should also be clear that an attempt to recreate this signal using a single pulse width would require a much larger number of pulses. With a single pulse width, as in the original FRI formulation, the narrowness of the QRS pulses would require a narrow width, and a greater number of narrow pulses would have to be summed to create the wider $\mathrm{T}$ and $\mathrm{S}$ pulses.

With seven pulses we require 14 complex numbers to represent the signal. This represents a reduction in the storage requirements from the 90 original sample values to 28 real numbers. However, it may be that a more significant aspect of this model is that it reduces the ECG signal to a small number of components that represent the main features of the signal. We expect to continue our investigations by seeking improvements to test simulations of ECG signals and determining whether the model parameters can be used for diagnostic purposes.

\section{REFERENCES}

[1] M. Vetterli, P. Marziliano, and T. Blu, "Sampling signals with finite rate of innovation," IEEE Trans. Signal Process., vol. 50, no. 6, pp. 1417-1428, 2002.

[2] T. Blu, P.-L. Dragotti, M. Vetterli, P. Marziliano, and L. Coulot, "Sparse sampling of signal innovations," IEEE SPMag, vol. 25, no. 2, 2008.

[3] R. Prony, "Essai experimental et analytique," J. Ecole. Polytech.(Paris), vol. 2, pp. 24-76, 1795.

[4] V.F. Pisarenko, "The retrieval of harmonics from a covariance function," Geophys. J., vol. 33, pp. 347-366, Sept. 1973.

[5] R. Roy and T. Kailath, "ESPRIT: Estimation of Signal Parameters Via Rotational Invariance Techniques," IEEE Trans. on ASSP, vol. 37, no. 7, pp. 984-995, July 1989.
[6] B. De Moor, "Total Least Squares for Affinely Structured Matrices and the Noise Realization Problem," IEEE Trans. on Signal Processing, vol. 42, no. 11, pp. 3104-3113, November 1994.

[7] J.A. Cadzow, "Signal enhancement-a composite property mapping algorithm," IEEE Trans. on ASSP, vol. 36, no. 1, pp. 49-62, Jan 1988.

[8] D.W. Tufts and R. Kumaresan, "Estimation of frequencies of multiple sinusoids: Making linear prediction perform like maximum likelihood," Proc. IEEE, Volume 70, p. 975-989, vol. 70, pp. 975-989, 1982.

[9] A. Hormati, "Sensing and recovery under sparsity constraints," Ph.D. thesis, EPFL, Lausanne, Switzerland, 2010

[10] Baechler, G., "Sensing ECG signals with variable pulse width finite rate of innovation," Master's Thesis, EPFL, Lausanne, Switzerland, in preparation.

[11] J. Pan and J. W. Tompkins, "A real-time QRS detection algorithm," IEEE Transactions on Biomedical Engineering, vol. BME-32, p. 230236, 1985. 